\title{
Magnet and Cryostat Configurations For a Multi-port Quadrupole Array*
}

\author{
M. A. Green, and R. O. Bangerter \\ Lawrence Berkeley National Laboratory \\ Berkeley CA 94720, USA
}

\begin{abstract}
August 2000
Presented at the Applied Superconductivity Conference 17 September through 22 September 2000, Virginia Beach VA, USA to be published in IEEE Transactions on Applied Superconductivity 11
\end{abstract}

* This work was performed the Lawrence Berkeley National Laboratory with the support of the Office of Science, United States Department of Energy under contract numbers DEAC03-76SF00098 


\title{
Magnet and Cryostat Configurations for a Multi-port Quadrupole Array*
}

\author{
M. A. Green, and R. O. Bangerter \\ Lawrence Berkeley National Laboratory, Berkeley CA, USA
}

\begin{abstract}
This report describes the results of a study of arrays of up to sixteen quadrupoles in a single cryostat surrounded by an induction accelerator that is used for accelerating high current heavy ion beams for fusion. Each quadrupole in the array can have a gradient of $72 \mathrm{~T}$ per meter, when the quadrupole has a warm bore diameter of $90 \mathrm{~mm}$. An array of sixteen quadrupoles can be made to fit into a round cryostat vacuum vessel with a diameter of $850 \mathrm{~mm}$. If the number of quadrupoles in the array is reduced to nine, the outer diameter of the cryostat is $700 \mathrm{~mm}$. It is proposed that the quadrupole array be conduction cooled using either a $4 \mathrm{~K}$ cryocooler or two phase liquid helium in pipes around the magnet array. The two-phase helium can be supplied to a string of multi-bore quadrupoles using a large refrigerator.
\end{abstract}

\section{INTRODUCTION}

An induction linac has been proposed to accelerate multiple high intensity heavy ion beams for heavy ion fusion. Quadrupoles are used to focus the high current beams as they are being accelerated. Since the current in any given beam is limited by space charge, it has been proposed that multiple beams be accelerated in order to produce the beam current desired. Since space is at a premium within an induction linac, multiple superconducting quadrupoles arranged in an array have been proposed to focus the multiple beams $[1,2,3,4]$. The superconducting quadrupoles should have a gradient of 50 to $100 \mathrm{~T}$ per meter depending on their warm bore beam aperture. The Lawrence Berkeley Laboratory has studied quadrupoles with both warm bores and cold bores. The apertures of the quadrupoles in arrays range from below $60 \mathrm{~mm}$ to over $120 \mathrm{~mm}$. Most of the quadrupole designs that have been studied have a peak induction of $4 \mathrm{~T}$. The highest induction within the coils, which affects the critical current of the superconductor, is about $5 \mathrm{~T}$. In most of the design studies to date, the superconducting coils are assumed to be made from niobium-titanium operating at $4.4 \mathrm{~K}$.

The design of a multiple bore quadrupoles and their cryostat is governed by the induction linac that will be built around the quadrupole array. The design of the quadrupole cryostat is governed by the following factors: 1) The radial distance from the outermost quadrupole to the outer wall of the cryostat should be minimized. 2) The external cryostat vacuum shell should be a circular cylinder, so that the electric field generated by the induction linac is not altered. 3) The longitudinal space allowed for cryogenic service, cold mass supports, and leads should also be minimized. 4) The radial

Manuscript received 18 September 2000.

* This Research is supported by the Office of Science US Department of Energy under contract number DE-AC0376SF00098 space between the superconducting quadrupole coils and the beam vacuum chamber should be minimized. 5) The number of beams in the quadrupole array should be optimized so that more beam current can be accelerated by a given size induction linac.

\section{SUPERCONDUCTING QUADRUPOLES DESIGNS}

There have been at least three different approaches that have been studied for quadrupole arrays for induction linac systems. The designs that have been considered for the quadrupole arrays fall into two broad general categories.

The first type of array design has the quadrupoles loosely spaced, so that the field from one quadrupole does not interact with neighboring quadrupoles (at least not very much). A loosely coupled array is illustrated in Figure1. Quadrupole arrays of this type often will have iron shielding between the quadrupoles[1]. In such arrays, the quadrupoles can be operated independently of each other. The downsides of such an array are the reduced number of quadrupoles for given cryostat diameter and the fact that more superconductor is needed to generate the quadrupole field.

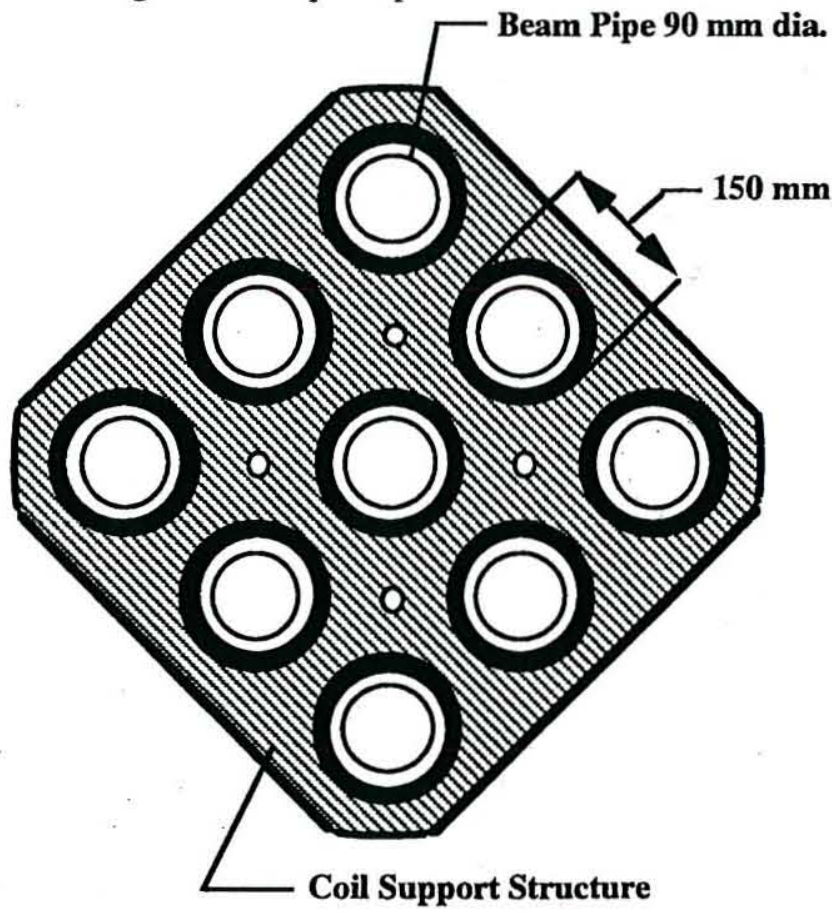

Figure 1. A Loosely Coupled Nine Quadrupole Diamond Array. The quadrupoles in the array have a $90-\mathrm{mm}$ warm bore. 


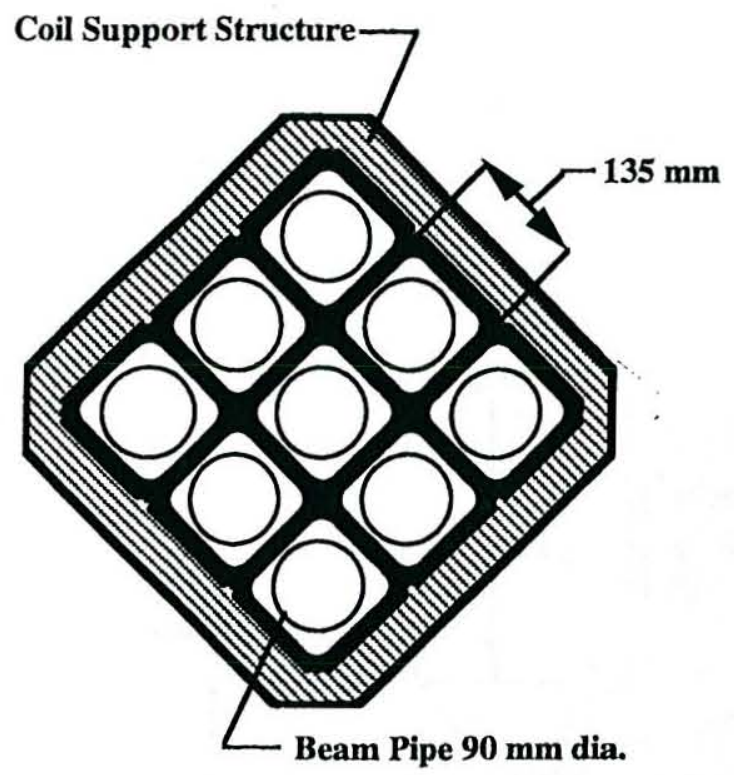

Figure 2. A Closely Coupled Nine Quadrupole Diamond Array. The quadrupoles in the array have a $90-\mathrm{mm}$ warm bore.

The second array design shown in Figure 2 has the quadrupoles closely packed. There are two advantages of this approach. The first advantage is that the array is more compact so that one should be able to accelerate more beam current (more beams) in a given cylindrical space. The second advantage is that the superconductor needed to generate the field in one quadrupole is reduced by almost a factor of two compared to an uncoupled quadrupole array. Fewer ampereturns in the quadrupole coils are also reflected as a lower stored energy per quadrupole bore in the closely packed array. The optimization of the quadrupole field quality in a closely packed array involves all of the coils in all of the quadrupoles.

Table 1 compares the physical parameters for the loosely packed quadrupole and closely packed 3 by 3 arrays shown in Figures 1 and 2. Table 2 compares the parameters for the closely packed 3 by 3 and 4 by 4 quadrupole arrays shown in Figures 2 and 3. (See Ref. [5] for more comparisons.)

Figures 1 and 2 show diamond shaped quadrupole arrays. The array shape can also be a square. For a loosely packed array, the shape of the array of almost no consequence, because there is little interaction between the quadrupoles in the array. On the other hand, a closely packed square array will have coils ends that are bent over the corners of the square. The centers for current of any given polarity in a square array are located at the center of the sides of the square. As a result, quadrupoles in a square array will be either all focusing or all defocusing[5]. A closely packed diamond array will be assembled from coils that are wound in a flat plane[4]. The current centers for any given polarity are located at the corners of the diamonds. As a result, diamond arrays ane mixed focusing and defocusing quadrupoles. Having both types of quadrupoles in the same array does not appear to complicate the beam dynamics of the accelerator System.

TABLE 1. A COMPARISON OF LOOSEL Y PACKED AND CLOSELY PACKED 3 BY 3 QUADRUPOLE ARRAYS

Parameter

Coil Shape

Bore Diameter (mm)

Pole Induction ( $\mathrm{T}$ )

Minimum Coil Radius (mm)

Quadrupole Gradient $\left(\mathrm{T} \mathrm{m}^{-1}\right)$

Quadrupole Magnetic Length (mm)

Length of the Cryostat (mm)

Array Structure Side Length (mm)

Cryostat Can Outer Diameter (mm)

Cryostat Height (mm)

Cryostat Neck Thickness (mm)

Cold Mass per Quad Array (kg)

Quadrupole Array Current (A)

Quadrupole Array Stored Energy (kJ)

Quadrupole Array Inductance (H)
Loose Close

Round Diamond

$90 \quad 90$

$4.0 \quad 4.0$

$60 \quad 55$

$66.7 \quad 72.7$

$\sim 500 \sim 515$

$700 \quad 700$

$605 \quad 480$

$850 \quad 700$

$1585 \quad 1310$

$110 \quad 110$

$480 \quad 260$

$1000 \quad 1000$

$\sim 400 \sim 260$

$\sim 0.80 \sim 0.52$

Note: All values of field, stored Energy are at the design current

TABLE 2. A COMPARISON OF CLOSELY PACKED 3 BY 3 AND 4 BY 4 QUADRUPOLE ARRAYS

Parameter

Coil Shape

Bore Diameter (mm)

Pole Induction ( $\mathrm{T}$ )

Minimum Coil Radius (mm)

Quadrupole Gradient $\left(\mathrm{T} \mathrm{m}^{-1}\right)$

Quadrupole Magnetic Length (mm)

Length of the Cryostat (mm)

Array Structure Side Length (mm)

Cryostat Can Outer Diameter (mm)

Cryostat Height (mm)

Cryostat Neck Thickness (mm)

Cold Mass per Quad Array (kg)

Quadrupole Array Current (A)

Quadrupole Array Stored Energy (kJ)

Quadrupole Array Inductance $(\mathrm{H})$

$\begin{array}{cc}3 \times 3 & 4 \times 4 \\ \text { Array } & \text { Array } \\ & \\ \text { Diamond } & \text { Diamond } \\ 90 & 90 \\ 4.0 & 4.0 \\ 55 & 55 \\ 72.7 & 72.7 \\ \sim 515 & \sim 515 \\ 700 & 700 \\ 480 & 605 \\ 700 & 850 \\ 1310 & 1585 \\ 110 & 110 \\ 260 & 350 \\ 1000 & 1000 \\ \sim 260 & \sim 480 \\ \sim 0.52 & \sim 0.96\end{array}$

Note: All values of field, stored Energy are at the design current

\section{QUADRUPOLE ARRAY CRYOSTAT}

Figure 3 shows a cross-section of the cryostat for a sixteen quadrupole (four by four) array with quadrupoles that have a $90-\mathrm{mm}$ warm bore. The cryostat shown in Figure 4 uses a tension band support system. The same cryostat could also use the vertical support-cylinder support system that has been proposed for solenoids that are within a high acceleration gradient induction linac[5,6]. Either of the cold mass support systems will fit in $110 \mathrm{~mm}$ of longitudinal space at the center of the quadrupole array. 


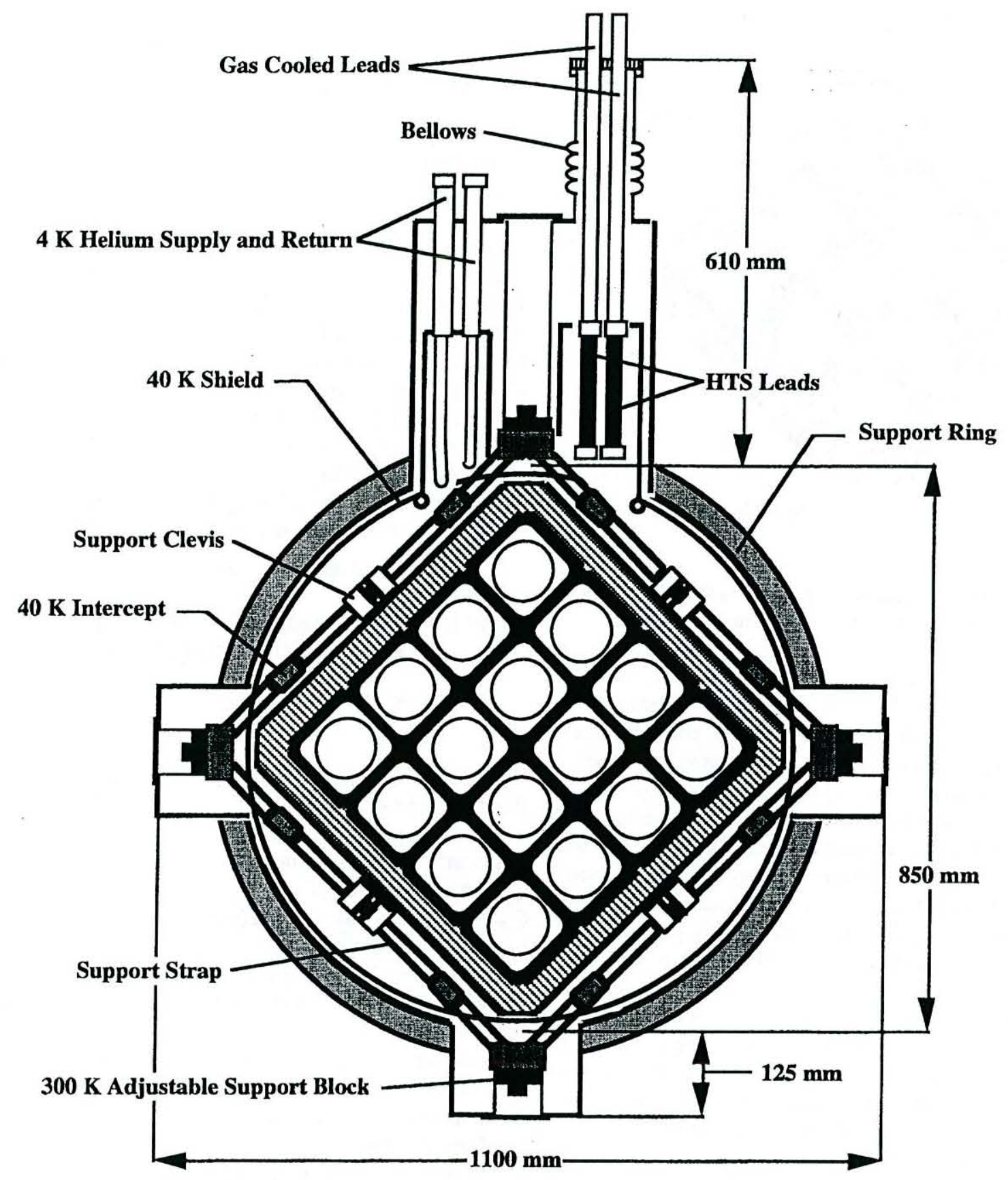

Figure 3. A Cross-section of a Sixteen Quadrupole Array with a Tension Band Cold Mass Supports, HTS Leads between $40 \mathrm{~K}$ and $4 \mathrm{~K}$, Gas Cooled Electrical Leads to $300 \mathrm{~K}$, and the $4 \mathrm{~K}$ and $40 \mathrm{~K}$ Cryogenic Services Port

The cold mass of the array shown in Figure 4 is about $350 \mathrm{~kg}$. The cold mass support is designed to withstand accelerations of four gee's in any direction. Not shown in Figure 4 is the longitudinal support, which is a pair carbonfiber hollow push-pull rods. The tension bands shown in Figure 4 can be made from either $\mathrm{E}$ glass or carbon fiber[7]. The HTS leads, gas cooled leads, and the cryogenic services from the refrigerator are all contained in the neck, which can be made $110 \mathrm{~mm}$ thick in the longitudinal direction.
Table 3 shows the heat flows into the sixteen-quadrupole array cryostat shown in Figure 3. From Table 3, it is clear that the largest $4.4 \mathrm{~K}$ heat load is down the 1000 -ampere high temperature superconductor (HTS) leads. Cooling within the $4.4 \mathrm{~K}$ region is by conduction from the various heat sources to a cooling tubes that carry two-phase helium at $4.4 \mathrm{~K}[8]$. As long as the quadrupole bores have 40 to $50 \mathrm{~K}$ shields, between the warm bore tube and the coils the temperature drop between the quadrupole superconducting coils and the 
two-phase helium cooling tube can be kept below $0.1 \mathrm{~K}$. A large heat flow to $4.4 \mathrm{~K}$ region in the magnet bore will require that there be liquid helium in the magnet bore.

\section{TABLE 3. CLOSELY PACKED FOUR BY FOUR QUADRUPOLE ARRAY HEAT LOADS}

Source of Heat

\begin{abstract}
Cold Mass Supports
Multi-layer Insulation

Helium Bayonet Tubes

Instrumentation Wires

1000 A Leads
\end{abstract}

Total Heat Flow

The heat load into the shield circuit is dominated by the heat flow through the multi-layer insulation. Half of the multi-layer insulation heat load in Table 3 comes from the sixteen $90-\mathrm{mm}$ warm quadrupole bores. The cooling for the shields comes from the helium refrigerator at a point in the high-pressure side of the heat exchanger string where the temperature is about $30 \mathrm{~K}$. Helium at $30 \mathrm{~K}$ from the refrigerator will be used to cool the shields, the cold mass thermal intercepts, and the $1000 \mathrm{~A}$ gas cooled leads that are connected to the HTS leads between $50 \mathrm{~K}$ and $4.4 \mathrm{~K}$. In order to cool the gas cooled leads between $50 \mathrm{~K}$ and $300 \mathrm{~K}$ a gas flow of about $0.11-\mathrm{g} \mathrm{s}^{-1}$ is required for the two leads. The temperature rise in the shield flow circuit, when the helium flow is $0.11 \mathrm{~g} \mathrm{~s}^{-1}$ for the $1000 \mathrm{~A}$ leads will be about $18 \mathrm{~K}$. The temperature at the top of the HTS leads will be about 50 $\mathrm{K}$. Using HTS leads and using gas from a point in the refrigerator at $30 \mathrm{~K}$ will reduce the overall refrigeration required to cool a string a quadrupole arrays[9].

\section{QUADRUPOLE ARRAY QUENCH PROTECTION}

In theory, even the sixteen $90-\mathrm{mm}$ warm bore quadrupole array can be protected using a simple dump resistor. At a current of $1000 \mathrm{~A}$, the $\mathrm{EJ}^{2}$ limit for the magnets is about $4.3 \times 10^{22} \mathrm{~A}^{2} \mathrm{~m}^{-4} \mathrm{~J}$. The $\mathrm{L}$ over $\mathrm{R}$ time constant for the dump circuit must be about 1.5 seconds. The problem with an active dump circuit is the need for a reliable quench detection system. An alternative approach is to shunt the quenching quadrupole array through cold diodes and balancing resistors that are in parallel with the array quadrupoles. This approach, which is completely passive, requires no quench detection circuit. '(Cold diodes and resistors in parallel with the coil are commonly used to protect NMR and MRI magnets.) Quenching of one quadrupole in an array of closely coupled quadrupoles such as those shown in Figure 2 or 3 will result in adjacent quadrupoles becoming normal through heat transfer from the quadrupole where the quench started. Quench back between dipoles is more difficult to achieve in a loosely packed array such as is shown in Figure 1.

\section{CONCLUDING COMMENTS}

A number of types of superconducting quadrupole designs have been proposed for a multi-beam heavy ion accelerator. Closely coupled quadrupoles in the array appear to be very attractive, provided one can design the array so that all of the quadrupoles produce the required good quality magnetic field.

A cryostat for a multiple bore quadrupole array reported here and in Reference 5 appears to be suitable for virtually all of the superconducting coil designs that have been proposed for the quadrupole arrays to date. Through the use of HTS current leads between $50 \mathrm{~K}$ and $4 . \mathrm{K}$, one can greatly reduce the refrigeration needed to cool the quadrupole array. Cooling the $40 \mathrm{~K}$ shields, the cold mass intercepts and the gas cooled leads between $50 \mathrm{~K}$ and $300 \mathrm{~K}$ with $30 \mathrm{~K}$ gas from the refrigerator will reduce the helium refrigeration needed to cool a long series of quadrupole arrays.

\section{ACKNOWLEDGEMENTS}

The authors acknowledge the many discussions with Peter Seidl and Andy Faltens of the Lawrence Berkeley National Laboratory concerning the design of quadrupole arrays and their cryostats.

\section{REFERENCES}

[1] S. Caspi, et al, "Superconducting Quadrupole Magnet Array for a Heavy Ion Fusion Driver," IEEE Transaction on Applied Superconductivity 9, No. 2, p 463, (1999)

[2] R. B. Meinke, et al, "Development of Quadrupole Arrays for Heavy-Ion Fusion," IEEE Transactions on Applied Superconductivity 10, No. 1 p 463, (2000)

[3] J. H. Schultz, et al, "Superconducting Quadrupoles for the Heavy Ion Fusion Integrated Research Experiment (IRE) and High Current Experiment (HCX)," these proceedings IEEE Transactions on Applied Superconductivity 11 (2001)

[4] N. Martovetski and R. Manahan, "Focusing Magnets for HIF Based on Racetracks." these proceedings IEEE Transactions on Applied Superconductivity 11 (2001)

[5] M. A. Green, "The Magnet Cryostats and the Cryogenic System for Multiple Bore Superconducting Quadrupoles Inside of an Induction Linac," Lawrence Berkeley National Laboratory report LBNL-46035, (2000)

[6] M. A. Green, et al, "A Thin Superconducting Solenoid for Use in a Phase Rotation Induction Linac," these proceedings IEEE Transactions on Applied Superconductivity 11 (2001)

[7] D. Evans, "Materials Technology for Magnet Insulation and Bonding," IEEE Transactions on Applied Superconductivity 10, No 1, p1300, (2000)

[8] M. A. Green, W. B. Burns and J. D. Taylor, "Forced TwoPhase Cooling of Large Superconducting Magnets," Advances in Cryogenic Engineering 25, p 271, Plenum Press, New York, (1979)

[9] M. A. Green, "The role of Superconductor in Reducing the Refrigeration Needed to Cool the Leads of a Superconducting Magnet", Cryogenics 30, No. 9, Supplement, p 679, (1990) 